\title{
Contractilidad Uterina y Frecuencia Cardiaca Fetal en la Preeclampsia Severa Durante el Primer Período del Parto (Periodo Dilatante)*
}

\author{
Dres.: R. Cifuentes B. ${ }^{* *}$, C.A. Quintero J.**
}

En oportunidad anterior (I) se publicaron los resultados del análisis de los cambios de la Contractilidad Uterina (C.U.) y la Frecuencia Cardíaca Fetal (F.C.F.) en la Preeclampsia severa durante el segundo período del parto (período expulsivo) en pacientes que no presentaron recién nacidos deprimidos ( $\mathrm{Ap}$ gar $>$ 7) productos de embarazo de termino ( $>38$ ss) con feto único $y$ en presentación de vértice. En este trabajo queremos analizar los parámetros anteriores desde el punto de vista de monitorización electrónica (C.U. y F.C.F.) en un grupo de embarazadas normales y con

* Con la ayuda financiera del Fondo Co. lombiano de Investigaciones Científicas y Proyectos Especiales “Francisco José de Caldas" (Colciencias), Proyecto 015-3-24 75.

** Laboratorio de Medicina Perinatal, Departamento de Obstetricia y Ginecología, Facultad de Salud, Universidad del Valle, Cali, Colombia. preeclampsia severa durante el primer período del parto (período dilatante).

\section{METODOS Y PACIENTES}

Se seleccionaron 40 pacientes əmbarazadas que ingresaron en el primer meríodo del parto (período dilatante) con feto único vivo y una edad gestacional de 38 a 41 semanas. Se tomaron 2 grupos de 20 pacientes cada uno, el primero sin ninguna patología asociada (grupo control) y el segundo con diagnóstico de preeclampsia severa (Presión Arterial diastólica mayor de $100 \mathrm{mmHg}$, edema y/o albuminuria).

Este último grupo recibió un tratamiento uniforme consistente en Sulfato de Magnesio a una dosis de 4 grs. iniciales y luego 1 gramo por hora en infusión continua, fenobarbital 200 mgs. IM cada 8 horas e hidralazina 5 mgrs IV cada 30 minutos cuando la T.A. diastólica fuese mayor de $110 \mathrm{mmHg}$. El grupo control no recibió ninguna medicación. 
El período dilatante de todas las pacientes fue de una duración normal de 6 a 10 horas y con un rango de dilatación de 4 a $10 \mathrm{cms}$. Los recién nacidos tuvieron un puntaje de Apgar de 7 o más al 5o. minuto de vida y la evolución en el período neonatal temprano (7 días) fue normal.

\section{MONITORIA DEL TRABAJO DE PARTO}

En ambos grupos se hizo registro continuo de la presión intrauterina mediante cateter transcervical conectado a un traductor de presión Bell Howell y éste a su vez a un monitor fetal Corometrics 111. Simultáneamente se registro la F.C.F. mediante registro directo, utilizando un electrodo de cloruro de plata colocado en el cuero cabelludo del feto (2).

\section{ESTUDIO CUANTITATIVO DE LA C.U.}

En todos los casos se cuantificaron los siguientes parámetros: intensidad $(\mathrm{mm} \mathrm{Hg}$, frecuencia) (número de contracciones en 10 minutos), actividad uterina (unidades Montevideo) y tono $(\mathrm{mmHg})$. Lo ante- rior se hizo para cada una de las contracciones desde $4 \mathrm{cms}$, de dilatación hasta la dilatación completa. Se compararon en ambos grupos utilizando el test de " $t$ " para muestras independientes.

\section{ESTUDIO CUANTITATIVO DE LA F.C.F.}

En todos los casos se analizó la F.C.F. basal y la variabilidad latido a latido de la línea de base, cuantificando antes de cada contracción el valor máximo y mínimo registrado en la F.C.F. basal y la diferencia entre ellas. Esta variabilidad así calculada se promedió para cada uno de los grupos estudiados. Además se analizaron los siguientes cambios periódicos: aceleraciones, desaceleraciones tempranas, (Dips I), desaceleraciones variables (Dips Umbilicales), desaceleraciones tardías (Dips Tipo II), y para cada uno de estos cambios se calculó su amplitud (latido por minuto) y duración (segundos). Se calculó la proporción de contracciones que producen cambios periódicos de la F.C.F. cuantificando el número total de ellos. Las variables analizadas fueron comparadas en ambos grupos por medio del test de " $\mathrm{t}$ " para muestras independientes.

Tabla No. 1

PARAMETROS DE LA CONTRACTILIDAD UTERINA EN LOS GRUPOS CONTROL Y CON PREECLAMPSIA SEVERA (PERIODO DILATANTE)

CONTROL $(n=812)$ PREECLAMPSIA $(n=717)$

\begin{tabular}{lcccccc}
\hline & $\bar{x}$ & E.S. & $\bar{x}$ & E.S. & $t$ & $\mathrm{p}$ \\
$\begin{array}{l}\text { INTENSIDAD } \\
\text { (mm de Hg) }\end{array}$ & 51.0 & 7.2 & 46.4 & 5.4 & 0.52 & N.S. \\
$\begin{array}{l}\text { FRECUENCIA } \\
(\text { (en 10 min.) }\end{array}$ & 4.2 & 0.3 & 3.8 & 0.5 & 0.72 & N.S. \\
$\begin{array}{l}\text { ACTIVIDAD UTERINA } \\
(\text { Un. Montev.) }\end{array}$ & 214.2 & 46.4 & 176.3 & 23.2 & 0.74 & N.S. \\
$\begin{array}{l}\text { TONO } \\
\text { (mm de Hg) }\end{array}$ & 3.2 & 1.4 & 7.4 & 1.4 & 2.20 & 0.05 \\
\hline
\end{tabular}




\section{RESULTADOS}

Contractilidad Uterina: La Tabla No. 1 nos enseña los cambios presentados en los diferentes parámetros estudiados: intensidad, frecuencia, actividad uterina $y$ tono para los dos grupos.

Los valores de $\mathrm{P}$ para los tres primeros no fueron estadísticamente significativos y para el tono que el valor de $P$ fue 0.05 y muestra diferencia significante sus valores tanto en el grupo de control como en las Preeclampsias severas estuvieron dentro de los límites normales $k 12$ $\mathrm{mmHg}$ ).

Cambios periodicos y basales de la F.C.F.

La Tabla No. 2 nos muestra los resultados encontrados para los diferentes parámetros, asi:

\section{Frecuencia Cardíaca Fetal Basal}

Fue de 143.9 para el grupo control y de 140.5 para los casos de preeclampsia,

Tabla No. 2

CAMBIOS EN LA LINEA DE BASE Y CAMBIOS PERIODICOS DE LA FRECUENCI.^ CARDIACA FETAL EN LA PREECLAMPSIA SEVERA DURANTE EL PRIMER PERIODO DEL PARTO (PERIODO DILATANTE)

\begin{tabular}{|c|c|c|c|c|c|}
\hline & & CONTROL & TOXEMIA & $t$ & $\mathrm{p}$ \\
\hline VARIABILIDAD & & $15.5 \pm 0.9$ & $9.9 \pm 2.3$ & 2.1 & 0.05 \\
\hline F.C.F. BASAL & & 143.9 & 140.5 & 0.4 & N.S. \\
\hline ASCENSOS & $\begin{array}{l}\text { Amplitud } \\
\text { Duración } \\
\text { Incidencia }\end{array}$ & $\begin{array}{l}18.0 \\
23.4 \\
32.4\end{array}$ & $\begin{array}{l}16.1 \\
34.3 \\
38.6\end{array}$ & $\begin{array}{l}0.4 \\
0.8 \\
1.1\end{array}$ & $\begin{array}{l}\text { N.S. } \\
\text { N.S. } \\
\text { N.S. }\end{array}$ \\
\hline $\begin{array}{l}\text { DESACELERACIONES } \\
\text { TEMPRANAS }\end{array}$ & $\begin{array}{l}\text { Amplitud } \\
\text { Duración } \\
\text { Incidencia }\end{array}$ & $\begin{array}{l}23.2 \\
23.5 \\
42.2 \pm 1.7\end{array}$ & $\begin{array}{l}16.5 \\
36.6 \\
23.5 \pm 1.6\end{array}$ & $\begin{array}{l}1.5 \\
1.7 \\
2.7\end{array}$ & $\begin{array}{l}\text { N.S. } \\
\text { N.S. } \\
0.02\end{array}$ \\
\hline $\begin{array}{l}\text { DESACELERACIONES } \\
\text { VARIABLES }\end{array}$ & $\begin{array}{l}\text { Amplitud } \\
\text { Duración } \\
\text { Incidencia }\end{array}$ & $\begin{array}{l}42.5 \\
43.5 \\
16.0^{ \pm}-1.6\end{array}$ & $\begin{array}{l}28.1 \\
48.9+ \\
44.0 \pm 2.9\end{array}$ & $\begin{array}{l}1.8 \\
0.5 \\
3.3\end{array}$ & $\begin{array}{l}\text { N.S. } \\
\text { N.S. } \\
0.001\end{array}$ \\
\hline $\begin{array}{l}\text { DESACELERACIONES } \\
\text { TARDIAS }\end{array}$ & $\begin{array}{l}\text { Amplitud } \\
\text { Duración } \\
\text { Incidencia }\end{array}$ & $\begin{array}{l}- \\
- \\
0\end{array}$ & $\begin{array}{r}24.8 \\
59.2 \\
2.4\end{array}$ & $\begin{array}{l}- \\
-\end{array}$ & \\
\hline
\end{tabular}


diferencias las cuales no tienen significancia estadística.

\section{Variabilidad de la F.C.F.}

El grupo control presentó una variabilidad de $15.5 \pm 0.9$ de la F.C.F. En el grupo con preeclampsia severo, la variabilidad fue de $9.9 \pm 2.3$. Esta diferencia mostró significancia estadística ( $p<$ 0.05, Fig. 1).

Figura No. 1

\section{VARIABILIDAD DE LA FRECUENCIA CARDIACA FETAL}

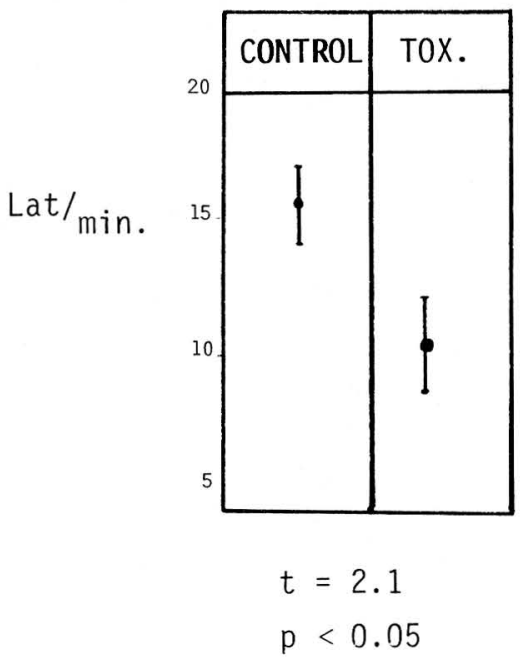

Fig. No. 1. Variabilidad de la línea de base de la FCF durante el período dilatante. Se observa como en el grupo con preeclampsia severa la variabilidad de la línea de base se encuentra significativamente disminuida con res. pecto a los controles.

\section{Aceleraciones}

No hubo diferencias estadísticamente significativas entre el grupo control (32. 4) $y$ el grupo de pacientes toxémicas (38.6).

\section{Desaceleraciones Tempranas}

En ambos grupos estudiados se encontró una proporción de desaceleraciones tempranas que fue significativa estacísticamente pues fue menor para el grupo de toxémicas $(23.5 \pm 1.6)$ que para el grupo control (42.2 \pm 1.7 , Fig. 2), no encontrando significancia en lo que se refiere a amplitud y duración de las desaceleraciones.

Figura No. 2

\section{DESACELERACIONES TEMPRANAS}

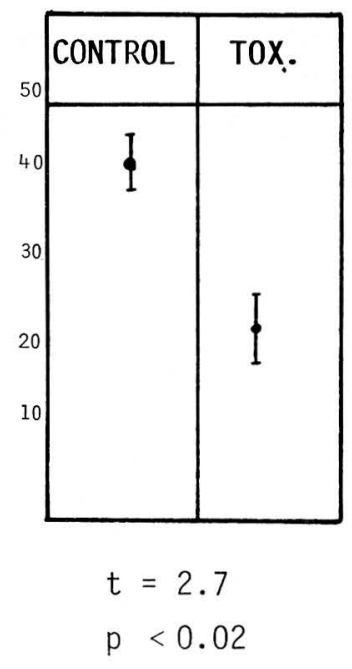

Fig. No. 2. Desaceleraciones tempranas (Dips tipo I o cefálicos). Puede verse que hay una proporción mayor de Dips tipo I en el grupo control que en el grupo de pacientes con preeclampsia severa durante el período dilatan. te (ver comentarios).

\section{Desaceleraciones variables}

En el grupo control la proporción de desaceleraciones variables fue de $16.0 \pm$ 1.6 mientras que en el grupo de preeclámpticas fue de $44 \pm 2.9$ con un valor 
de $P<0.001$, diferencia la cual es estadísticamente significativa, Fig. 3. No encontramos diferencia estadística entre los dos grupos en cuanto se refiere a la amplitud y a la duración.

Figura No. 3

\section{DESACELERACIONES VARIABLES}

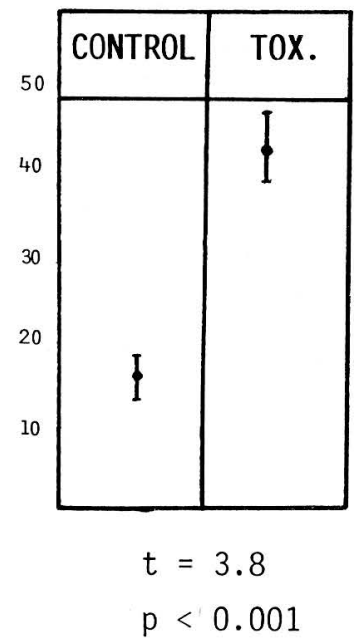

Fig. No. 3. Desaceleraciones variables (Dips variables o umbilicales). Se observa claramente como el grupo de pacientes con preeclampsia presenta una proporción de Dips variables sig. nificativamente mayor que el grupo control durante el período dilatante.

\section{Desaceleraciones tardías}

No se encontraron Dips II o desaceleraciones tardías en el grupo control como era de esperar y un $2.4 \%$ en el grupo de toxémicas, aunque en todos los casos fueron ocasionales y $\sin$ criterio de severidad.

\section{DISCUSION Y COMENTARIOS}

\section{Contractilidad Uterina}

El análisis de la contractilidad uterina hecho en un grupo de pacientes toxémicas y comparado con un grupo control, nos ha mostrado que durante el período dilatante las condiciones de la misma son sensiblemente iguales, encontrando sólo una significancia estadística en el tono, el cual fue mayor en el grupo de las toxémicas, pero sin salirse de los límites de la normalidad según ha sido descrito (3).

Como se hizo el comentario en artículo previo, el tratamiento establecido especialmente a base de Sulfato de Magnesio pudo haber influido en los cambios de la contractilidad uterina en el sentido de disminuir la intensidad de las contracciones, lo cual hace que no sean claramente diferenciables en el período dilatante, como sí lo son durante el embarazo (4).

F.C.F.: La línea de base de la FCF no mostró diferencia en los dos grupos estudiados.

La variabilidad latido a latido mostró diferencias estadísticamente significantes entre el grupo control y el grupo de toxémicas, en las cuales hubo disminución de la variabilidad; sin embargo, no podemos atribuir este fenómeno como un efecto del Sulfato de Magnesio sobre la variabilidad de la FCF de corto y largo plazo (5).

\section{Aceleraciones}

No hubo diferencias estad ísticamente significativas para los dos grupos, comosí se encontraron en el estudio del segundo período del parto, en el cual fueron mayores en el grupo de toxémicas que en el grupo control. El hecho descrito de que en los embarazos de alto riesgo y como en nuestro caso, las toxémicas, se presente una disminución de las aceleraciones tanto las que siguen a movimientos fetales como las periódicas, no lo muestran nuestros resultados; puede posiblemente deberse a que el número de 
casos es pequeño o a que haya una explicación fisiopatológica diferente.

\section{Desaceleraciones tempranas}

Los Dips Tipo I o desaceleraciones tempranas fueron un hallazgo frecuente en ambos grupos como era de esperar cuando se monitorizan paciente durante el primer período del parto y con membranas rotas tal como se describe en otros trabajos previos (6). Sin embargo, no encontramos ninguna explicación al hecho de que fueron más frecuentes en el grupo control que en las toxémicas.

\section{Desaceleraciones variables}

Los fenómenos atribuibles a compresión del cordón umbilical fueron más frecuentes en las pacientes toxémicas que en el grupo control, diferencia la cual nos habla de un fenómeno patológico atribuible a la disminución del volúmen del líquido amniótico, como ha sido descrito anteriormente (7). Es importante hacer notar que los fenómenos dependientes de la compresión del cordón, como los que se presentan en la toxemia del embarazo, pueden ser más frecuentes aún que aquellos dependientes de insuficiencia uteroplacentaria (desaceleraciones tardías o Dips Tipo II) evidencia ya encontrada $y$ publicada por otros autores (8).

\section{RESUMEN}

Se hace una revisión de un grupo de pacientes con preeclampsia severa y su equivalente número de controles normales con el fin de estudiar los cambios de la contractilidad uterina y la frecuencia cardíaca fetal durante el primer período del parto (período dilatante). De este grupo de 40 pacientes ya se publicaron los hallazgos de la revisión de los mismos parámetros durante el período expulsivo y su relación con morbilidad perinatal (1). En todas se realizó registro continuo de C.U. y F.C.F.

Se encontró diferencia significativa en la variabilidad de la línea de base de la FCF en el sentido de una disminución en las pacientes toxémicas. También fue notorio el hallazgo de una mayor proporción de desaceleraciones variables. (Dips umbilicales o variables) en los fetos de madres toxémicas que en aquellos de los controles y se discute la fisiopatología de los cambios anteriores.

Es importante hacer referencia al hecho de que los cambios dependientes de la compresión del cordón umbilical son más frecuentes que aquellos dependientes de una insuficiencia útero-placentaria en la preeclampsia severa durante el primer período del parto.

\section{UTERINE CONTRACTILITY AND FETAL HEARTH RATE IN SEVERE PREECLAMPSIA DURING THE FIRST PERIOD OF LABOR}

\section{Summary}

We made a revision in a group of patients with severe preeclampsia and the equivalente number of normal pregnancies. to study the changes in uterine contractility (U.C.) and Fetal Hearth Rate (FHR) during the first period of labor. From this group of 40 patients were published the changes during the second period of labor and its relation with perinatal morbility (1). All of them had direct and continous monitoring of U.C. and FHR. We found decreased variability if base line of FHR and a greater incidence of variable decelerations (umbilical dips) in tetus of mothers with severe preeclampsia as compared with fetuses from normal mothers. We discussed the possible physiopathological mechanisms of these changes. 
Finally is important to note FHR changes which depend of umbilical card are more frequently than those of uterus

\section{BIBLIOGRAFIA}

1. CIFUENTES, R., Quintero, C. Contractilidad Uterina y F.C.F. en la preeclamp: sia severa durante el período expulsivo del trabajo de parto. Rev. Col. Obst. Gin. 33, 317, 1982.

2. HON, F. An Atlas of FHR Patterns New Haven, Harty Press, 1968.

3. ALVAREZ, H., CALDEYRO-BARCIA, R. Fisiopatología de la contractilidad uterina $y$ sus aplicaciones en la Clínica Obstétrica. Maternidad e Infancia, 1954.

4. СОВО, E. Uterine hipercontractility in toxemia of pregnancy. Am. J. Obstet. Gynecol: 98:505, 1964. placental insufficiency in the severe Preeclampsia during the first period of labor.

5. BABAKNIA, A., NIEBYE, J.R. The effect of Magnesium Sulfate on FHR baseline variability. Obst. Gynecol. 51 (Supl): 2, 1978.

6. CALDEYRO-BARCIA, R. SCHWARCZ, R.L., ALTHABE, O.: Effects of rupture of membrane on fetal heart rate patterns. Int. J. Gynaec. 10:169, 1972.

7. GADD, R.L. The volume of liquor ammii in normal and abnormal pregnancies. $\mathrm{J}$. Obst. Gynecol. Br. Commonw. 73:11, 1966.

8. СОВО, E. Toxemia del embarazo en Obstetricia. Eds. R. Schwarcz, C. Duverges y A.G. Díaz. 3ra. edición. EI Ateneo, Buenos Aires, 1983. 